\title{
O DESIGN E NOVAS TECNOLOGIAS PARA O AVANÇO DAS PRÓTESES: EXPLORAÇÃO, INOVAÇÃO E AVALIAÇÃO
}

\author{
Gabriel de Souza Prim \\ Universidade Federal de Santa Catarina \\ gabrielsprim@gmail.com \\ Milton Luiz Horn Vieira \\ Universidade Federal de Santa Catarina \\ milton.vieira@ufsc.br
}

Resumo: As novas tecnologias oferecem uma ampla gama de produtos e serviços para a promoção em saúde. O foco do presente projeto de pesquisa está nas próteses, utilizando novas tecnologias para o aprimoramento destes produtos, pois observa-se que novas tecnologias apresentam oportunidades para testes e avaliações de peças e componentes. Este trabalho delimita um método para explorar a experiência dos usuários com próteses transtibiais e procura associar as tecnologias de Captura de Movimentos, Impressão 3D e Scanner 3D para aprimoramento das próteses, possibilitando o desenvolvimento de um novo produto a ser validado por métodos de medição de equilíbrio. $O$ presente trabalho explora a experiência dos usuários com próteses transtibiais e procura associar as tecnologias apontadas para o aprimoramento dos produtos existentes nesta área, com validação em método de medição de equilíbrio. A tecnologia de Scanner 3D possibilita a geração de modelos virtuais com grande percentual de automação, minimizando o trabalho manual, tornando o processo de digitalização tridimensional de peças mais dinâmico, permitindo alteração de peças existentes em um espaço de tempo menor e com maior confiabilidade quando comparado com os processos manuais. As máquinas de prototipagem rápida, também conhecidas como Impressoras 3D, permitem que as peças scanneadas possam ser modificadas digitalmente e prototipadas para testes em curto espaço de tempo. Os principais fatores motivadores deste método são sua relevância social e sua não trivialidade. A relevância do método para a sociedade emerge a partir do momento que os resultados alcançados forem capazes de melhorar a qualidade de vida de usuários de prótese, enquanto sua não trivialidade é notada ao perceber que os resultados esperados com esse método possuem capacidade de oferecer aprimoramentos e/ou novos produtos cruciais que podem não ter sido observados até hoje por falta de equipamentos apropriados. Objetiva-se com este método explorar e avaliar o desenvolvimento e aprimoramento de próteses com o auxílio das tecnologias mencionadas, identificando os produtos que podem ser melhorados e identificando quais deles são compatíveis com a tecnologia 
de impressão 3D, pois a impressão 3D possui algumas limitações tecnológicas que precisam ser levadas em consideração para o presente projeto de pesquisa. Após estas identificações, analisa-se soluções e verifica-se a solução apontada. A pesquisa proposta é de natureza exploratória e divide-se em Identificação, Análise, Geração de Ideias e Verificação. A etapa de Identificação possui o objetivo de apontar os produtos destinados ao público alvo da pesquisa; A etapa de Análise é destinada a investigação dos problemas apontados com maior profundidade; A etapa de Geração de Ideias busca propor soluções para os problemas observados na fase de Análise; A etapa de Verificação destinase para a comprovação das soluções apontadas pela geração de ideias. Espera-se que esta pesquisa ofereça uma visão sobre os problemas enfrentados no cotidiano nas pessoas amputadas, indicando soluções para os problemas que afetam a segurança e o conforto desse público. Esperase também fomentar os estudos relacionados com o desenvolvimento de produtos assistivos, prototipação rápida, ergonomia dentre outros tópicos com publicações nacionais e internacionais nas revistas científicas pertinentes.

Palavras-chave: Tecnologia Assistiva, Próteses, Impressão 3D, Scanner 3D, Motion Capture.

\section{INTRODUÇÃO}

O contexto da amputação, de modo geral, trata-se de um processo traumático para o indivíduo, buscando se estabilizar psicologicamente e socialmente diante de um momento crítico na vida do indivíduo. O sucesso da reabilitação é imprescindível para a independência do indivíduo para a realização de uma marcha equilibrada e das atividades cotidianas (BOCCOLINI, 2000; CARVALHO, 2003).

Assim, entende-se que a manutenção do equilíbrio é o meio de avaliação de eficiência de próteses. Segundo MACHADO (1993), para haver a manutenção do equilíbrio é necessária a integridade dos elementos anatômicos e funcionais, que compreendem o aparelho vestibular, a visão, os centros nervosos, sistema proprioceptivo e o sistema musculoesquelético. As próteses buscam devolver ao indivíduo amputado a integridade dos elementos anatômicos e funcionais. $O$ indivíduo amputado de membro inferior pode apresentar dificuldades na manutenção do equilíbrio estático, o que pode gerar quedas, e consequentemente fraturas (BARAÚNA ET AL., 2006).

O presente trabalho apresenta-se como um método para pesquisa e desenvolvimento em próteses utilizando um conjunto de tecnologias utilizadas em design. Trata-se da medição do equilíbrio de usuários de prótese, utilizando métodos associados com Motion Capture. Motion Capture é o processo de captura dos movimentos de um objeto real e transferir a informação para o meio digital. Este processo é utilizado em diversas áreas como a medicina e a robótica.

Em conjunto com a tecnologia de Motion Capture, observa-se que novas tecnologias apresentam oportunidades para testes e avaliações de peças e 
componentes. Nos últimos anos, as máquinas de prototipagem rápida, também conhecidas como Impressoras 3D, apresentam-se com máquinas mais baratas e menores. Tais máquinas podem apresentar grande utilidade em prototipagem e design. A tecnologia "open source" está acessível a qualquer pessoa com acesso a internet. Atualmente, existem diversas tecnologias de impressão 3D. Todas as tecnologias se baseiam no princípio de executar diversos fatiamentos da figura, obtendo uma fina camada da figura que é impressa através do processo de deposição de materiais das partes sólidas da figura, obtendo o objeto desejado sobrepondo as diversas camadas uma sobre a outra (TAKAGAKI, 2012).

Outra tecnologias vêm emergindo nos últimos anos, como por exemplo os Scanner 3D. A criação de modelos tridimensionais semelhantes à realidade é uma tarefa que pode ser facilitada com a utilização de alguns métodos, entre eles o escaneamento em 3D, que possibilita a geração de modelos virtuais com grande percentual de automação, minimizando o trabalho manual (PERES, 2013). A aquisição de dados tridimensionais de maneira automatizada tem diversas aplicações, destacando-se na indústria mecânica para o desenvolvimento de projetos por meio de engenharia reversa e para a verificação da precisão de peças fabricadas a partir de modelos digitais (CELANI E CANCHERINI, 2009).

Nesse contexto, o método defendido neste trabalho delimita-se a explorar a experiência dos usuários com próteses transtibiais e procura associar essas tecnologias para aprimoramento dos produtos existentes nesta área, possivelmente desenvolver um novo produto para este público a serem validados no método de medição defendido na pesquisa desenvolvida no mestrado do autor deste projeto.

\section{DESENVOLVIMENTO}

Antes de apresentar o método (sua natureza e suas etapas), faz-se necessária uma fundamentação teórica das tecnologias utilizadas.

\subsection{Motion Capture}

O Sistema de Motion Capture (MoCap) é um meio de oferecer coordenadas tridimensionais de um objeto a partir de um conjunto de imagens bidimensionais captadas por um conjunto de câmeras dispostas ao redor do ator captado (PARK, 2013). Criado primeiramente para aplicações na medicina, o motion capture óptico consiste em um sistema de câmeras, como a da figura 1, controladas por um computador.

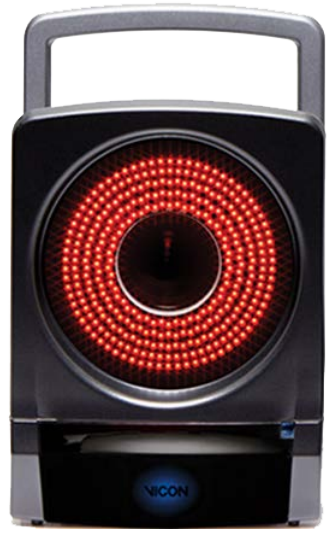

Figura 1 Câmera de Motion Capture Vicon T-40S.

Fonte: Vicon, 2015. 
$\mathrm{O}$ ator ou objeto a ter seus movimentos capturados possui marcadores, como da figura 2, fixados em seu corpo, podendo estes ser reflexivos ou emissivos e denominados, respectivamente, passivos e ativos (KITAGAWA, 2008).

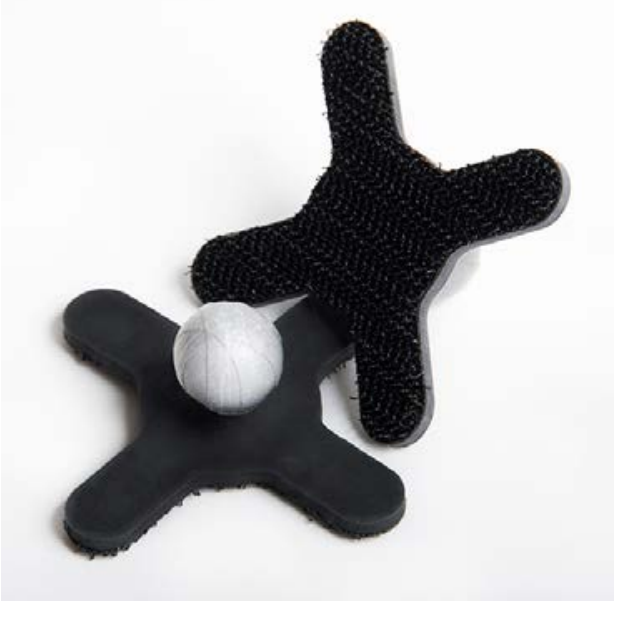

Figura 2 Marcadores Reflexivos.

Fonte: MoCap Solutions. Integrated Base Motion Capture Markers. Disponível em: <

http://mocapsolutions.com/integrated-base-motion-capture-markers/" Acessado em: 15/11/2015.

Criado primeiramente para aplicações na medicina, o Motion Capture óptico consiste em um sistema de câmeras controladas por um computador. O sujeito a ter seus movimentos capturados possui marcadores fixados em seu corpo.

As câmeras utilizadas atualmente possuem características técnicas que permitem a captura de movimentos com resposta em tempo real e são equipadas com infravermelho, resolvendo qualquer problema com a iluminação do espaço onde o equipamento é instalado, garantindo a confiabilidade dos dados (Vicon, 2015).

\subsection{Impressão 3D}

A impressão 3D está inserida num conceito denominado RP (Rapid Prototyping) ou prototipação rápida, onde procuramos criar uma peça a partir de um desenho, conforme apresentado na Figura 3. Atualmente, existem diversas tecnologias de impressão 3D.

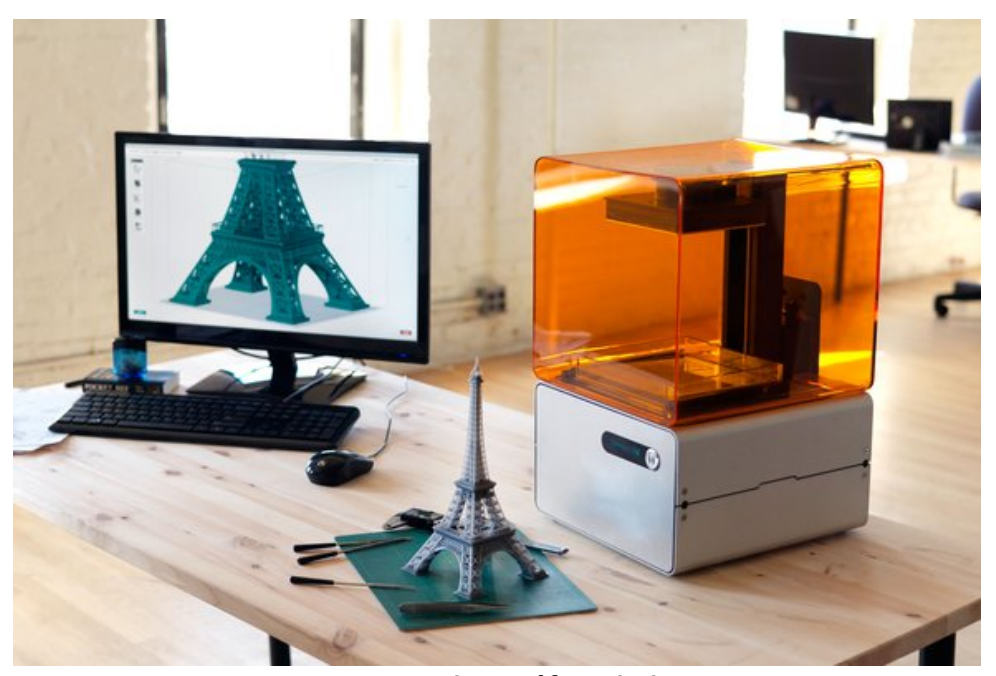

Figura 3 Impressora 3D estereolitográfica de baixo custo. Fonte: FormLabs. Disponível na internet por http em:< http://formlabs.com/> Acessado em: 10/01/2016. 
Todas as tecnologias se baseiam no princípio de executar diversos fatiamentos da figura, geralmente na horizontal, obtendo uma fina camada da figura que é impressa através do processo de deposição de materiais das partes sólidas da figura. Sobrepondo as diversas camadas uma sobre a outra, obtemos o objeto final desejado (TAKAGAKI, 2012). Existem diversas maneiras de depositar diferentes materiais em camadas. Pode-se citar os seguintes sistemas:

- $\quad$ Stereolithography (SLA)

- $\quad$ Selective Laser Sintering (SLS)

- $\quad$ Direct Metal Laser Sintering (DMLS)

- $\quad$ Selective Laser Melting (SLM)

- $\quad$ Fused Deposition Modelling (FDM)

- $\quad$ Digital Light Processing (DLP)

- Multi-jet Modelling (MJ M)

- $\quad$ Plastic Sheet Lamination

Apesar dos diversos sistemas, os processos atuais estão baseados em alguns procedimentos em comum (TAKAGAKI, 2012). A impressão 3D possui algumas limitações tecnológicas que precisam ser levadas em consideração para o presente projeto de pesquisa. Por utilizarem deposição de múltiplas e finas camadas, o tempo demandado para gerar um objeto de pequenas dimensões é bastante considerável. Outra limitação apontada por TAKAGAKI (2012) é que os desenhos tenham pontes, impedindo que sejam executados diretamente.

A utilização desta tecnologia em fabricação em série de peças ainda não se mostra viável, pois os custos e eficiência do processo são questionáveis, principalmente pelo fator do tempo de fabricação de uma peça (TAKAGAKI, 2012).

\subsection{Scanner 3D}

Existem diversos tipos de scanners tridimensionais conhecidos, com vantagens e desvantagens para cada tipo de aplicação. Em linhas gerais, um scanner 3D pode ser classificado como scanners com contato e scanners sem contato. Os scanner com contato se caracterizam como scanner com contato físico com o objeto, com alta precisão demandando maior tempo de aquisição de dados. Os scanner sem contato físico, como o da figura 4, demandam menor tempo para aquisição de dados, com menor precisão quando comparado com os scanners sem contato (PERES, 2013).

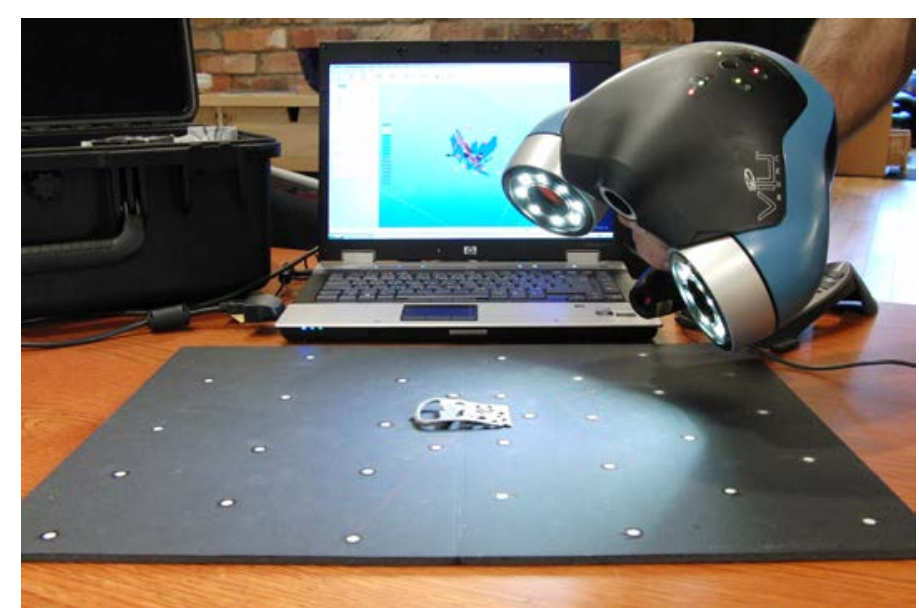

Figura 4 Scanner 3D sem Contato.

Fonte: CreativeTools.se - VIUscan - Laser-scanned - ZPrinter - 3D printed - Viking Belt Buckle 24.

Disponível na internet por http em: <https://www.flickr.com/photos/creative_tools/4530599701/> 


\subsection{Metodologia Proposta}

O método apresentado é destinado à pesquisa de natureza exploratória, conforme classificação de Gil (2008), pois tem como objetivo principal conhecer mais a respeito da área de produtos destinados a indivíduos amputados no Brasil.

A pesquisa se divide em Identificação, Análise, Geração de Ideias e Verificação. A etapa de Identificação possui o objetivo de apontar os produtos destinados ao público alvo da pesquisa: indivíduos amputados. Esta etapa se propõe a explorar as próteses com mais profundidade, identificando suas peças, quais as vantagens e desvantagens de cada uma. As próteses modulares são próteses compostas por diversas peças, que são escolhidas conforme as necessidades do usuário. Esta etapa busca encontrar problemas que precisam de solução economicamente vantajosas para o desenvolvimento dessas peças.

Com o objetivo de analisar os problemas apontados na etapa anterior, a etapa de análise é destinada a investigação dos problemas apontados com maior profundidade, apontando os fatores limitantes para o problema ainda não ter sido solucionado. Esta etapa também contempla a viabilidade econômica das soluções atuais. Durante as pesquisas anteriores na área, o autor deste projeto observou que o custo das próteses é um fator que limita o acesso das próteses às pessoas menos favorecidas economicamente, enquanto o Sistema Único de Saúde (SUS) não consegue arcar com os custos de próteses modulares apropriadas, dependendo de tipos de próteses defasados com desempenho insatisfatório.

A etapa de Geração de Ideias busca propor soluções para os problemas analisados na etapa anterior. Aqui, as tecnologias de escaneamento 3D e impressão 3D permitirão a exploração do design utilizado atualmente e prototipação de possíveis soluções para os problemas encontrados. Aqui também busca-se soluções com desempenho semelhante, diante de um custo de produção menor.

As ideias geradas na etapa anterior devem apontar solução para os problemas investigados. A etapa de verificação é destinada a comparabilidade da solução apresentada com o produto utilizado comumente no mercado. A comparação será com base nos resultados em testes de equilíbrio executados por voluntários diante um sistema de captura de movimentos. Os voluntários devem realizar os testes com sua prótese e, em seguida, realizar os mesmos testes com a solução encontrada, prototipada com a impressão 3D.

\section{CONCLUSÃO}

Espera-se que o método apresentado ofereça uma visão sobre os problemas enfrentados no cotidiano nas pessoas amputadas, indicando soluções para os problemas que afetam a segurança e o conforto desse público alvo. Espera-se ainda fomentar os estudos relacionados com o desenvolvimento de produtos assistivos, prototipação rápida, ergonomia dentre outros tópicos com publicações nacionais e internacionais nas revistas científicas pertinentes.

\section{REFERÊNCIAS}

ASSUMPÇÃO, T.N. Órteses - Princípios Básicos, in Freitas, P.P (Ed). Reabilitação da Mão. Atheneu, pp. 27, 2005. 
BADKE, M. B. et. al. (2011) Tongue-based biofeedback for balance in stroke: results of an 8-week pilot study. Archives of Physical Medicine and Rehabilitation, Vol. 92 No. 9, pp. 1364-1370.

BARAÚNA, M. A. et. al., (2006) Avaliação do equilíbrio estático em indivíduos amputados de membros inferiores através da biofotogrametria computadorizada, Brasilian Journal of Physical Therapy, Vol. 10 No. 1, pp. 83-90, 2006.

BARAÚNA M. A. et. al. Avaliação do equilíbrio estático do portador de diabetes mellitus pela biofotogrametria. Brazilian Journal of Physical Therapy, Vol. 7 No. 1, pp. $57-62,2003$.

BERG, K. $\mathrm{O}$ et. al. Measuring balance in the elderly: validation of an instrument. Canadian Journal of Public Health, Vol. 83 No. 2, pp. S7 - S11, 1992.

BOCCOLINI, F. Reabilitação: amputados, amputações e próteses, 2nd ed., São Paulo, 2000.

CARVALHO, J. A. Amputações em membros inferiores: em busca da plena reabilitação. Manole, São Paulo, 1999.

CARMELI, E.; IMAM, B. Health promotion and disease prevention strategies in older adults with intellectual and developmental disabilities. Front. Public Health, Vol. 2. No. 31. pp. 1-7, 2014.

CELANI, G; E CANCHERINI, L. Digitalização tridimensional de objetos: um estudo de caso. Anais do Sigradi 2009 p.309-311. São Paulo, 2009.

CONESA, L. et. al. An observational report of intensive robotic and manual gait training in sub-acute stroke. Journal of NeuroEngineering and Rehabilitation, Vol. 9, No. 13, pp. 1-9, 2012.

EMILIO, J. et. al. The association of flexibility, balance, and lumbar strength with balance ability: risk of falls in older adults. Journal of Sports Science and Medicine, Vol. 13, pp. 349-357, 2014.

GIL, Antonio Carlos. Métodos e Técnicas de Pesquisa Social. 6.ed. São Paulo: Atlas, 2008

GUERREIRO, J.; PINTO, $\mathrm{H}$. The engaged university and institutional changes in portuguese innovation systems. Int. J. Transitions and Innovation Systems, Vol. 2, No. 1, pp. 72-90, 2012.

ICKENSTEIN, G. W., et. al. Static posturography in aging and Parkinson's disease', Frontiers in Aging Neuroscience. Vol. 4, No. 20, pp. 1-7, 2012.

KIM, S.; YUK, G.; GAK, H.. Effects of the horse riding simulator and ball exercises on balance of the elderly. J. Phys. Ther. Sci., Vol. 25, No. 11, pp. 1425-1428, 2013.

KITAGAWA, M. AND WINDSOR, B. Mocap for Artists: Workflow and Techniques for Motion Capture. 1a ed., Elsevier, Oxford, 2008.

KOVÁCS, É. et. al. Effects of a multimodal exercise program on balance, functional mobility and fall risk in older adults with cognitive impairment: a randomized controlled single-blind study. Eur. J. Phys. Rehabil. Med., Vol. 49, No. 5, pp. 639-648, 2013. 
LEE, K. J. et. al. The effects of a balance exercise program for enhancement of gait function on temporal and spatial gait parameters in young people with intellectual disabilities. J. Phys. Ther. Sci. Vol. 26, No. 4, pp. 513-516, 2014.

MACHADO, A. B. M. Neuroanatomia Funcional, 2nd ed., Atheneu, Rio de Janeiro, 1993.

MIYAMOTO, S.T et al. Brazilian version of the Berg balance scale. Brazilian Journal of Medical and Biological. Vol. 37, pp. 1411-1421, 2004.

PARK, H. S. et. al. A new position measurement system using a motion-capture camera for wind tunnel tests. Sensors, Vol. 13, No. 1, pp. 12329-12344, 2013.

PERES, F. O.. Scanner 3D: Problemas e Soluções. Trabalho de Conclusão de Curso (Graduação). Bacharelado em Ciência da Computação - Universidade Estadual de Londrina, 2013.

RAMOS, A. R.; SALLES, I. C. D. Fisioterapia: aspectos clínicos e práticos da reabilitação, Aspectos clínicos in: Borges, D., Moura, E. W., Lima, E. and Silva, P. A. C. (Eds.), Artes Médicas, São Paulo, 234-262, 2005.

RODRIGUES, A. V. N., CAVALCANTI, A.; GALVÃO, C. Órtese e prótese, in: Cavalcanti, A. and Galvão, C. (Eds.), Terapia Ocupacional: fundamentação \& prática, Rio de Janeiro, pp. $435-450,2007$.

SANGLARD, R.C.F. et. al. A influência do isostretching nas alterações do equilíbrio em idosos. Revista Brasileira Ciência e Movimento, Vol. 15 No. 2, pp.63 - 71, 2007.

WHITNEY, S.L., POOLE, J.L.; CASS, S.P. A review of balance instruments for older adults. American Journal of Occupational Therapy, Vol. 52, pp. 666-671, 1998.

World Health Organization (WHO). (2011) Health technology assessment of medical devices. Medical Device Technical Series, Geneva. Disponível na internet por http em: $<$ http://whqlibdoc.who.int/publications/2011/9789241501361 eng.pdf > Acesso em 02 ago. 2015.

Vicon. What is Motion Capture?. Disponivel na internet por http em: <http://www.vicon.com/what-is-motion-capture> Acessado em 02 Ago. 2015. 